\title{
ANÁLISIS
}

\section{LA APORTACIÓN DE VALOR EN TIEMPOS DE CRISIS}

\author{
Josep-Maria Rodríguez-Rovira
}

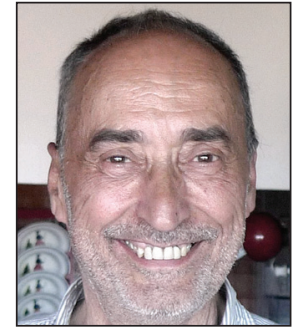

Josep-Maria Rodríguez-Rovira, ingeniero técnico industrial, tiene 25 años de experiencia en el sector de la información. Es cofundador de la empresa Doc6 y fue su consejero delegado durante el período 1988-2011.

rodriguezrovira@gmail.com

\section{Resumen}

Se ofrece el punto de vista de un profesional del sector sobre la trayectoria de los profesionales de la información, documentación y biblioteconomía, abordando las tendencias de los años más recientes y, sobre todo, las perspectivas de futuro, teniendo presente la actual situación de crisis y algunas de sus consecuencias. Se propone como el núcleo central del futuro de la profesión la gestión del conocimiento y su papel fundamental en los procesos de innovación, debido a su proyección profesional, económica, cultural y social.

\section{Palabras clave}

Valor añadido, Gestión del conocimiento, Innovación, Profesión, Futuro, Sector información, Biblioteconomía y documentación.

\section{Title: Adding value in times of crisis}

\section{Abstract}

The viewpoint of an experienced professional on the career paths in the field of library and information science is presented, addressing the trends in recent years and especially the future prospects. The current economic crisis and some of its consequences are taken into account. Given their professional, economic, cultural and social importance, knowledge management and its critical role in innovation processes are proposed as being at the core of the profession's future.

\section{Keywords}

Added values, Knowledge management, Innovation, Profession, Future, Information sector, Library and information science.

Rodríguez-Rovira, Josep-Maria (2013). "La aportación de valor en tiempos de crisis". El profesional de la información, mayo-junio, v. 22, n. 4, pp. 333-338.

http://dx.doi.org/10.3145/epi.2013.jul.09

\section{Venimos de lejos...}

En la sección egipcia del Louvre hay dos piezas a las que tengo un gran aprecio, porque para mí simbolizan dos aspectos esenciales del desarrollo de la sociedad a lo largo de la historia y en el futuro. Una, es una estela de Tot, el dios del conocimiento, de la escritura y de los escribas, el de los bibliotecarios de la época. Es muy elocuente que ya en el antiguo Egipto se unieran de manera tan clara el conocimiento y los bibliotecarios. La otra es una imagen de Akenatón, el faraón heterodoxo, el innovador que quiso cambiar el culto de Amón por el de Atón, una revolución que estremeció el Egipto de la época.
A mi entender forman parte de una simbología que une conocimiento e innovación, entendidas como voluntad de cambio positivo, de avance, que se retroalimentan y forman un conjunto imprescindible en los avances de la humanidad.

Desde la prehistoria, cuando la transmisión del conocimiento era casi exclusivamente oral y presencial y, a partir del desarrollo de la escritura, el conocimiento no ha cesado de avanzar, y también lo han hecho los medios para su extensión y difusión. La escritura posibilitó su difusión entre las personas de diferentes lugares. Comportó también otro avance importantísimo; la posibilidad de acumular conocimiento y hacerlo accesible a cualquiera que supiera leer. 
Nacieron las bibliotecas como centros de acumulación y difusión del saber. Pero no sólo esto...

\section{César lo tenía claro}

Muy pronto, algunos se dieron cuenta de que las bibliotecas, podían jugar un papel trascendente social y políticamente y también económicamente.

Como muy bien explican Fernández-Uriel y RodríguezValcárcel (2006), después de la guerra civil Julio César se planteó la creación de una biblioteca pública en Roma, la primera, como instrumento de prestigio y expansión de su influencia social y política, seguramente impulsado por dos importantes precedentes: la biblioteca de Alejandría fundada por los Tolomeos y conocida por él durante su estancia en Egipto el 48 a.C., y la de Pérgamo.

Su muerte le impidió llevar a cabo el proyecto, pero la semilla estaba plantada y finalmente Asinio Polión lo llevo a término unos años después.

\section{Libros y bibliotecarios}

Es conocido el proceso de evolución de los libros, sea en formato tablillas, papiro, rollos..., como receptáculos del conocimiento e instrumento de su difusión. Los bibliotecarios han jugado un papel decisivo como calígrafos y "escribidores" en los sucesivos soportes. Y como conocedores y transmisores del conocimiento de los contenidos a los lectores y a los copiadores monacales en la edad media.

La imprenta de Gutenberg fue revolucionaria porque permitió la difusión universal del conocimiento a gran velocidad y a costes cada vez más bajos, lo cual confirió a los bibliotecarios un papel mucho más relevante, aunque dentro de las limitaciones que imponían los medios de comunicación y transporte de la época.

Al cabo de varios siglos, a los libros se incorporaron la prensa y las revistas, y más tarde la radio, el teléfono y la televisión... Es un proceso de innovación permanente, en el que los medios materiales son, por requerimientos de la propia evolución del conocimiento, más y más sofisticados, más complejos, más fáciles de difundir y con mayor capacidad de penetración en la sociedad.

\section{Asociados al conocimiento. El meta conocimiento}

A lo largo de siglos los bibliotecarios-documentalistas han estado siempre vinculados a los diferentes soportes, formando parte de los procesos de creación, clasificación, localización y difusión del conocimiento.

En cada ámbito en que desarrollan su actividad -tanto en bibliotecas e instituciones públicas, como en centros privados, o en los universitarios y de investigación- el objetivo de los bibliotecarios-documentalistas es facilitar a sus usuarios el mayor conocimiento posible para el estudio, investigación, trabajo productivo o para los servicios a los ciudadanos. Son pues fundamentales creadores y gestores del meta conocimiento, esto es, de los conocimientos sobre el conocimiento.
Sin duda ésta es la profesión que ha tenido unos cambios más rápidos y profundos en menos tiempo. En los últimos 20 ó 30 años se ha pasado de gestionar las bibliotecas y la documentación con fichas de papel, a la informatización, a las bases de datos, a la integración de diferentes fuentes y recursos, a la edición electrónica, a internet, el uso de las redes sociales y la formación de los usuarios en las nuevas tecnologías. Los profesionales han tenido no sólo una adaptación rápida y entusiasta, sino que han jugado un papel impulsor en todo el proceso, con una clara voluntad de ofrecer el mejor servicio a los usuarios, conscientes de la proyección y trascendencia social y en todos los ámbitos, de su actividad.

Su adaptación no ha sido sólo técnica, sino conceptual, con asunción práctica de lo que comporta un nuevo paradigma en el tratamiento y difusión de la información y el conocimiento.

Se trata ahora de dar nuevos pasos adelante con una ampliación de objetivos, en cuanto a actividades a desarrollar y en cuanto a proyección profesional y social.

\section{Las crisis y sus retos}

Podemos decir sin temor a exagerar que estamos en una encrucijada de civilizaciones, tal vez en una crisis de la civilización occidental y euro céntrica tal como se ha caracterizado en los últimos siglos.

La globalización económica impulsada desde los valores de beneficio rápido y de concentración de riqueza y poder, ha comportado una expansión a todo el mundo de los centros de producción, con un desplazamiento, desde países como España, a países menos desarrollados, aprovechando el desfase industrial para obtener condiciones más ventajosas para las multinacionales y grandes consorcios; salarios y condiciones laborales inferiores, restricciones de derechos democráticos y laborales, legislaciones tolerantes con sus intereses, falta de exigencias en medio ambiente, etc.

La globalización ha acelerado también otro efecto previsible y con un gran potencial transformador para el futuro: la entrada de numerosos países en los procesos de innovación y desarrollo del conocimiento.

Esto comporta varios efectos:

- Importante avance de algunos países, sobre todo de los conocidos como BRICS (Brasil, Rusia, India, China, Sudáfrica) en la innovación y el desarrollo técnico-científico, del conocimiento, con la pérdida del monopolio de los países que lo han detentado en los últimos decenios.

- Pérdida de exclusividad de los centros de poder económico, y por ende político y militar de los países que lo han ejercido en los últimos siglos.

- Mayor competencia, con una presión a la baja de las condiciones laborales y socio-culturales de los países, como España, de desarrollo medio.

Todo lo enunciado comportará una resituación de recursos, de riqueza y poder que influirá en todos los aspectos de la vida. No cabe duda que esto puede provocar un aumento de las tensiones y que lo que podemos calificar de lucha por la vida se intensificará. 
En este contexto, uno de los elementos clave será la capacidad de uso del conocimiento y la innovación y los recursos que se destinen a ello. Porque el conocimiento permite una mayor eficiencia y por tanto también una mayor capacidad de supervivencia en todas las actividades, así como unas mayores posibilidades de proyección y de generar influencia en diferentes entornos y países.

España no tiene ni tendrá las cosas fáciles: a la falta de políticas de desarrollo industrial, hay que añadir los elevados endeudamientos públicos y privados, los devastadores efectos de la economía sumergida y la evasión fiscal, además de la corrupción y comportamientos irresponsables, cuando no delictivos, de numerosos políticos y empresarios.

A los recortes salariales y de derechos sociales se añaden los relacionados con la cultura y también con las políticas y recursos en investigación e innovación.

Los profesionales tenemos responsabilidades a las que no debemos renunciar, poniendo en valor nuestros conocimientos y experiencia profesional para que contribuyan a la eficiencia de las organizaciones en las que trabajamos, porque el interés y la forma en que ejercemos nuestra profesión tiene siempre una proyección económico-social y cultural.

\section{L@s documentalistas aportamos valor a la sociedad}

\section{Creamos valor}

La puesta en práctica de la capacidad de trabajo intelectual que tenemos los profesionales, crea un valor de uso para las organizaciones para las que trabajamos, que es tanto mayor cuanto mayores son nuestros conocimientos, nuestra experiencia, nuestra implicación, nuestra capacidad de relación interpersonal y de gestión emocional para el trabajo en equipo y la identificación con los objetivos de la organización.

En la situación actual de desarrollo tecnológico-industrial, el conocimiento se constituye como una fuerza productiva directa, de tal manera que las organizaciones que lo poseen disponen de un intangible que aporta valor a todas sus actividades.

Ya Marx en los Grundrisse (1857-1858), señala que "El conocimiento o knowledge social general se ha convertido en una fuerza productiva inmediata".

Esta es la profesión que más puede aportar en la gestión del conocimiento y en su difusión, porque de su formación, su cultura profesional y su experiencia colectiva se infiere que dispone de los instrumentos intelectuales, técnicos y metodológicos y del saber social necesarios para ello.

Es más, podemos decir que es la única profesión que puede hacerlo con garantías. Otras profesiones pueden hacer incursiones concretas en este ámbito, pero ninguna otra posee el corpus de conocimientos y experiencia que poseen los documentalistas.
Hace falta que los propios miembros de la profesión sean plenamente conscientes de ello y actúen en consecuencia. Esta profesión tiene, todavía, unas ciertas reminiscencias de "auxiliar" de otras, y de vocacional en el sentido de "ayudar" y no se trata de ayudar, si no de aportar, de contribuir.

Para ello, es condición imprescindible que desde la propia profesión y desde los centros educativos, en concreto las universidades, se asuman estos planteamientos y objetivos y se transmitan a los profesionales en formación. Es necesario que todos tengamos clara la visión global de nuestra capacidad y nuestras aportaciones a la sociedad, cada uno desde el ámbito de su actividad.

Debemos ver el conjunto del "bosque" de nuestra aportación científica-intelectual-profesional-social y no dejarnos deslumbrar por la continuada sucesión de "árboles", esto es, las herramientas que aparecen continuamente asociadas a los avances técnicos. Dominar buscadores, integradores, editores, redes sociales, etc., es imprescindible, pero situados en su papel de instrumentos de trabajo que sirven al objetivo principal: la gestión del conocimiento en el ámbito en que nos encontremos. Y aquí es posible y necesario aplicar métodos y servicios que puedan servir para que nuestro trabajo aporte valor a las organizaciones para las que trabajamos y, por extensión, a toda la sociedad.

\section{Sobre la empleabilidad}

En el estudio de Tejada y Moreiro (2003) sobre "Mercado de trabajo en biblioteconomía y documentación" se constata una tendencia hacia el aumento de la contratación por parte de las empresas, en contraste con las administraciones públicas, que ha sido el sector tradicional de creación de ocupación.

Para abordar el futuro más próximo, consideramos una valiosa aportación el artículo de Abadal, Borrego y Serra (2012), sobre "Mercado laboral de los profesionales de la información: evolución de la oferta y de los perfiles ocupacionales", en el que se hace una comparativa entre las solicitudes de empleo recibidas en la Facultat de Biblioteconomia i Documentació de la Universitat de Barcelona, en los períodos 2009-2010 y 2011-2012. Resaltamos algunos de los datos:

En el período 2009-2010, las ofertas de trabajo procedentes del sector público fueron el $49,9 \%$ y del sector privado un $50,1 \%$, de un total de 367 .

En cambio en el $2011-2012$, fueron de $13,3 \%$ y $86,7 \%$ respectivamente, de un total de 608 .

Respecto a los perfiles solicitados, el cambio es importantísimo:

\begin{tabular}{|l|c|c|}
\hline & $\begin{array}{c}\mathbf{2 0 0 9 - 2 0 1 0} \\
\%\end{array}$ & $\begin{array}{c}\mathbf{2 0 1 1 - 2 0 1 2} \\
\%\end{array}$ \\
\hline Biblioteconomía y documentación & 62,1 & 26,8 \\
\hline Archivos & 14,4 & 4,1 \\
\hline Marketing y comunicación & 6,0 & 32,7 \\
\hline Gestión de contenidos digitales & 6,6 & 25,7 \\
\hline Gestión cultural y educación & 7,4 & 7,2 \\
\hline Otros & 3,6 & 3,5 \\
\hline
\end{tabular}


Del análisis de las cifras hacen algunas consideraciones:

- La primera y más importante es que, aun en la situación de crisis, la oferta de empleo no sólo no decae, sino que aumenta.

- En pocos años se pasa de un predominio de la oferta pública de empleo, a una paridad y, muy rápidamente, a un predomino del sector privado.

- En las solicitudes de empleo en el sector privado tienen un peso fundamental las relacionadas con los sectores de marketing y comunicación y gestión de contenidos digitales.

- Otra conclusión importante es que los solicitantes no buscaban una licenciatura o diplomatura especifica de documentación-biblioteconomía, si no licenciados o diplomados en general.

Lo previsible es que estas tendencias se acentúen en el futuro y que todas las industrias relacionadas con la información y el conocimiento se desarrollarán extraordinariamente, puesto que condicionan la innovación como motor de avance fundamental en el desarrollo de las sociedades.

Debe tenerse presente que con internet y especialmente con los buscadores y las redes sociales, ha aparecido un fenómeno nuevo que se amplía vertiginosamente, en cuanto a posibilidades de información y comunicación.

Todo ello configura un panorama en el que aparecen posibilidades reales de empleo, y que éste deriva hacia el sector privado y concretamente en tecnologías de la información y gestión del conocimiento en términos amplios, y que los licenciados y diplomados en documentación tienen que competir por el puesto de trabajo con los de otras carreras. En esta competición con otros profesionales, los documentalistas partimos con una doble ventaja:

- Tenemos por formación una especialización en instrumentos, tanto de acceso a fuentes, como de integración y análisis, y de gestión y difusión de la información y del conocimiento, que no tienen otros profesionales.

- Tenemos también un importante entorno cultural-profesional con una gran tradición y una estructura organizativa (colegios y asociaciones, jornadas y congresos).

Todo ello nos da unas claras ventajas en la práctica profesional y, por tanto en la contratación. Digamos que en el famoso "hinterland" (zona alrededor) de Cronin et al., (2003), referenciado en el artículo que comentamos ${ }^{1}$, los documentalistas llevamos una importante ventaja sobre otros profesionales, en ámbitos de actividad que debemos considerar como propios y actuar en consecuencia.

No quiero cerrar este punto sin hacer referencia a dos aspectos que me parecen fundamentales cara a la actividad de futuro:

1) Relacionada con las universidades y centros de investigación.

En ellos las tareas y responsabilidades de los documentalistas se ha ido ampliando y van apareciendo más y más posibilidades. Una fundamental es la participación de los documentalistas como miembros de pleno derecho en los equipos de investigación asumiendo tareas de gestión de la información y del conocimiento, con responsabilidades específicas en el equipo y no sólo de soporte, a petición de resto de investigadores del equipo.

Sobre este tema Camón, Rey y Balagué (2012) publicaron un valioso artículo: "El apoyo a la investigación en las bibliotecas universitarias catalanas: estado actual".

2) Relacionada con las bibliotecas públicas y, específicamente, en su papel como dinamizadoras de la actividad económica y agentes de integración cultural-social.

Mediante servicios de información para las industrias y comercios de su área geográfica.

Con la organización de actividades e iniciativas que tiendan a una difusión cultural para realizar una tarea de integración social entre los sectores con mayor peligro de marginación y entre la población inmigrante.

Lo dicho implica que la formación, muy concretamente la formación universitaria de los profesionales, sea multifacética y aborde, además de los temas técnico-profesionales, los de gestión y relación, y que los colegios y las asociaciones profesionales jueguen un papel muy activo.

Esta profesión tiene la vocación de "ayudar", pero no se trata de ayudar, si no de aportar, de contribuir

\section{Panorámica de futuro}

Hemos hablado ya de los efectos de la globalización y de la crisis y de la necesidad de hacerles frente desde unos valores de formación intensiva, rigor profesional, eficiencia y compromiso con la proyección social de nuestro trabajo.

Las herramientas, los procesos, las metodologías de gestión del conocimiento conocerán un crecimiento exponencial, porque las confrontaciones entre países y zonas del planeta, tienen en la innovación uno de los campos de competencia.

La aportación de valor que comportará el aumento de la masividad mundial en el uso de buscadores y redes sociales, dará lugar a un incremento de la competencia entre los diferentes productos que ya existen y la creación de otros nuevos.

Debe tenerse en cuenta que el valor añadido fundamental de este tipo de servicios proviene de los propios usuarios, mediante la información que incorporan en las actividades que realizan en la Red y la atención que dedican a consultar información y a expresar sus opiniones.

En un reciente seminario celebrado 15 de mayo de 2013, por el Aula Rubió i Balaguer, en la Facultad de Biblioteconomia i Documentació de la $U B$, se abordó la situación de los principales buscadores y redes sociales en el mundo. Tal como expresó Lluís Codina, las actividades que realizan los usuarios en los buscadores y redes sociales, comporta la visualización de todo tipo de informaciones y anuncios que incorporan los administradores de portales y redes a sus sistemas. Es lo que éstos venden a las agencias comerciales 
y de marketing, y que también facilitan a los respectivos gobiernos y sus agencias, a la vez que contribuyen a difundir determinadas culturas y valores proclives a los intereses de sus gestores. Todo ello da lugar, además de a un fabuloso negocio, a una influencia cultural, política y aun geoestratégica.

Éstas son las razones de fondo que explican las fuertes pugnas entre buscadores de diferentes ámbitos de influencia, Google en EUA y parte de Europa (con una audiencia del $90 \%$ de internautas), Yandex en Rusia y toda la zona de la antigua Unión Soviética, incluida la parte asiática (con una audiencia del $60 \%$ de internautas, frente al $22 \%$ de Google), y Baidu en China y su área de influencia (con una audiencia del $85 \%$, frente a un $15 \%$ de Google). Hay que decir que China es el primer país del mundo en internautas, con el $39,9 \%$ de la población internauta del mundo.

De la importancia de estos procesos nos habla también Ignacio Ramonet, director de Le monde diplomatique versión en español, mayo de 2013. Nos cuenta que en el informe presentado al Presidente Obama, después de su toma de posesión en enero pasado, por el NIC (National Intelligence Council) de EUA, se alude al importante papel que realizarán las comunidades congregadas y vinculadas entre sí por internet y las redes sociales.

La generalización del acceso a la Red y la universalización en el uso de las nuevas tecnologías darán una gran movilidad a las acciones sociales, pero, a la vez, proporcionarán a los gobiernos unas posibilidades sin precedentes de controlar a sus ciudadanos, a la vez que modificarán todos los usos comerciales y la innovación y la actividad científica.

\section{Un tejido profesional-empresarial}

Otro sector de actividad que debemos resaltar, y que puede ser cada vez más importante, es el de la creación de empresas específicas para estas actividades, que actúen como consultoras colaborando con empresas y administraciones, aportando conocimientos y especialización.

La evolución de los segmentos de actividad profesional, cada vez con mayor peso de las empresas, está creando las condiciones para que haya un nicho de mercado cada vez más amplio y especializado.

Las empresas existentes en el sector han generado una cantidad notable de puestos de trabajo y esta tendencia aumentará en el futuro, a medida que empresas y administraciones constaten los resultados positivos que les pueden aportar los profesionales.

Es claro que estas actividades deben realizarse desde el planteamiento empresarial y que, por tanto deben tener unos costes que, sin duda, tendrán un retorno (ROI) ampliamente beneficioso para aquellos que contraten sus servicios.

Este ha sido un sector en el que la procedencia histórica, de trabajo y empleos, fundamentalmente de las administraciones, ha creado una cierta filosofía de gratuidad en todo tipo de prestación de servicios. Y esta gratuidad que debe seguir manteniéndose para los servicios prestados desde las entidades públicas, no debe pretenderse de las empresas, cuyos costes de inversión y actividad deben financiarse por sus clientes.

También debe resaltarse la importancia de que las administraciones y sus organismos se mantengan al margen de realizar actividades que podemos calificar de intrusismo en el sector, bien sea con la realización de trabajos de consultoría o con publicaciones especializadas, como ha sucedido en algunos casos en los años pasados, todo ello realizando una competencia desleal a las empresas, con el fin de obtener fuentes de financiación heterodoxas, contraviniendo en algunos casos a sus propios fines institucionales.

Permítaseme acabar con una llamada a la confianza en que, a pesar de la gravedad de los problemas actuales, y de que el futuro plantea muchísimas incógnitas y una profunda transformación del mundo, el trabajo de cada uno de nosotros como profesionales y de todos trabajando como colectivo, pueden sin duda contribuir al desarrollo de una sociedad mas equilibrada, más solidaria, más justa y humana.

\section{Nota}

1. Copiamos de BiD:

“Cronin (1993) utiliza el término hinterland para definir aquella capa de mercado laboral que envuelve el núcleo (heartland) tradicional de las bibliotecas y otras unidades de información".

\section{Bibliografía}

Abadal, Ernest; Miralpeix, Concepció (1999). “La enseñanza de la Biblioteconomía y la Documentación en la universidad española a finales de los noventa". Biblioteca i documentació, març, n. 2.

http://www.ub.edu/bid/02abamir.htm

Abadal, Ernest; Borrego, Àngel; Serra-Pérez, Rafael (2012). "Mercat laboral dels professionals de la informació: evolució de l'oferta i dels perfils ocupacionals. BiD, n. 29, dic. htttp://www.ub.edu/bid/29/abadal1.htm

Berardi, Franco (2003). La fábrica de la infelicidad. Nuevas forma de trabajo y movimiento global. Madrid: Traficantes de sueños.

Camón, Enric; Rey, Carina; Balagué, Núria (2012). “El apoyo a la investigación en las bibliotecas universitarias catalanas: estado actual". BiD, n. 29, dic.

http://www.ub.edu/bid/29/camon2.htm

Capitalismo cognitivo, propiedad intelectual y creación colectiva. Madrid: Traficantes de sueños, 2004.

Cercadors a internet i xarxes socials: Tendències de futur: Una visió més enllà de Google. Mayo 2013.

http://www.ub.edu/biblio/AJRB.html

Cronin, Blaise; Stiffler, Michael; Day, Dorothy (1993). "The emergent market for information professionals: educational opportunities and implications". Library trends, v. 42, n. 2, Fall, p. 257-276.

https://www.ideals.illinois.edu/bitstream/handle/2142/7899/ librarytrendsv42i2e_opt.pdf

Drucker, Peter (1999). Peter Drucker on knowledge worker productivity. 
http://www.knowledgeworkerperformance.com/PeterDrucker-Knowledge-Worker-Productivity.aspx

Edvinsson, Leif; Kivikas, Mart (2004). "La nueva perspectiva para la creación de valor". Revista de contabilidad y dirección, v. 1, pp. 47-57.

http://www.accid.org/revista/documents/intangibles_ castellano 047-057.pdf

Fernández-Uriel, Pilar; Rodríguez-Valcárcel, José A. (2006). "Julio César y la idea de biblioteca pública en la Roma antigua". Antigüedad y cristianismo, n. 23.

http://revistas.um.es/ayc/article/view/52451

III Jornada de biblioteca pública e inmigración. Bibliotecas públicas de Catalunya. Febrero 2010.

http://goo.gl/wzHLG,5

Marx, Karl. Elementos fundamentales para la crítica de la economía política (Grundrisse der Kritik der Politischen Ökonomie) 1857-1858. Vol. 2. México. Siglo XXI Editores, 2009, pp. 216-230.
Meroño-Cerdán, Ángel-Luis; Sabater-Sánchez, Ramón (2002). "Creación de valor empresarial a través del capital intelectual y la gestión del conocimiento". Gestión: Revista de economía, n. 21, pp. 18-25.

http://dialnet.unirioja.es/servlet/articulo?codigo $=2112712$

Miñarro-Yanini, María-Dolores (2002). La biblioteca pública como espacio de integración ciudadana. La biblioteca en la trinchera: problemas y oportunidades de la biblioteca pública ante la inmigración. Biblioteca Pública Municipal.

http://travesia.mcu.es/portalnb/jspui/bitstream/10421/1182/1/ com_249.pdf

National Intelligence Council (2012). Global trends 2030: Alternative worlds.

http://www.dni.gov/index.php/about/organization/ national-intelligence-council-global-trends

Ramonet, Ignacio (2013). “Editorial: El mundo en 2030". Le monde diplomatique. Edición en español, mayo. http://www.rebelion.org/noticias/2013/5/167645.pdf

\section{Anuario ThinkEPI 2013}

272 páginas de análisis de tendencias en información, documentación y comunicación

\section{Formulario de compra:}

http://www. thinkepi.net/pedido

\section{Información y pedidos:}

Isabel Olea

epi.iolea@gmail.com

+34-608491521

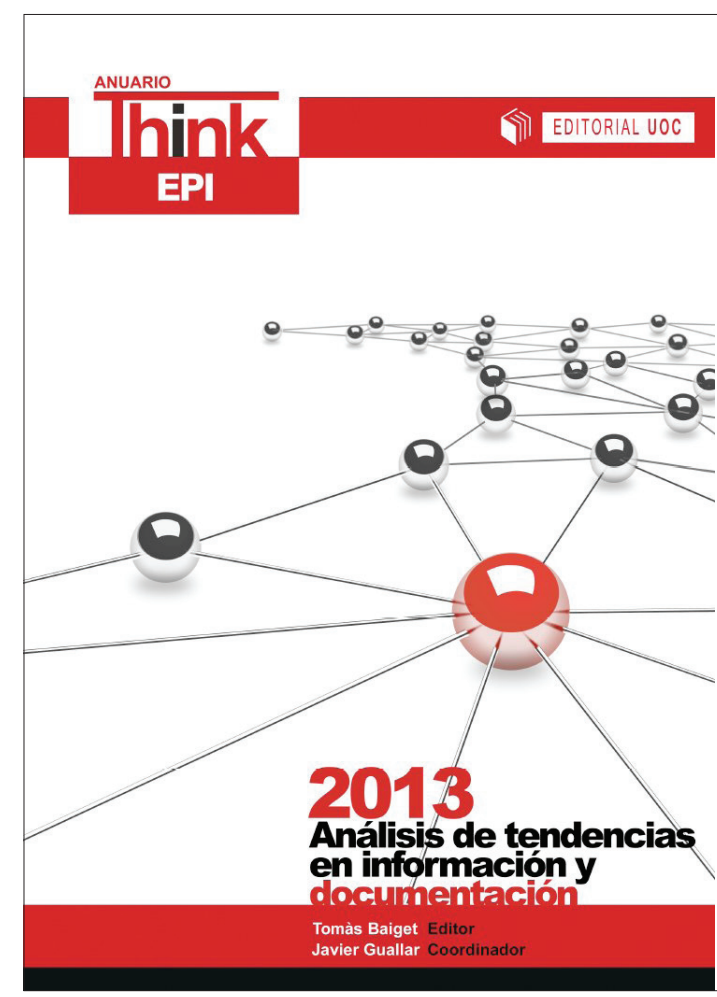

